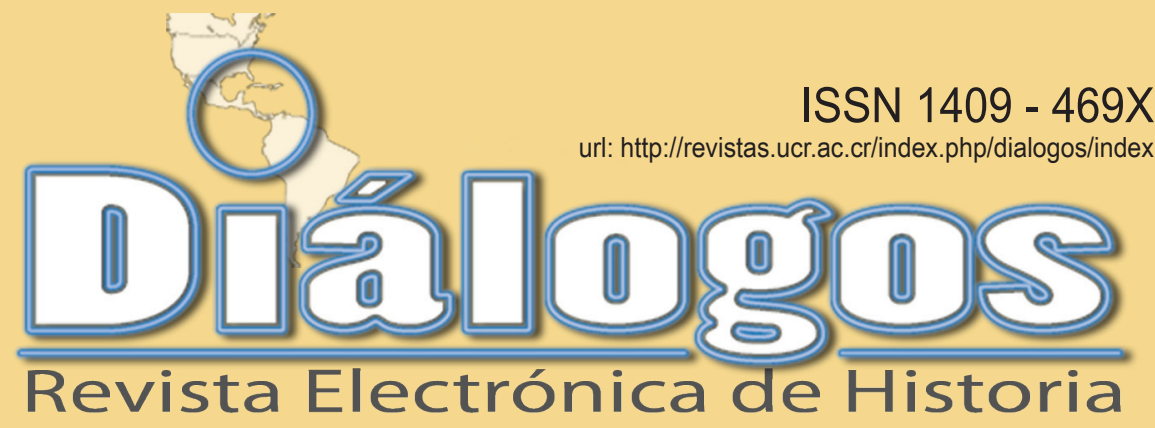

Escuela de Historia. Universidad de Costa Rica Volumen 15 Especial Región Occidente de Costa Rica - Octubre, 2014

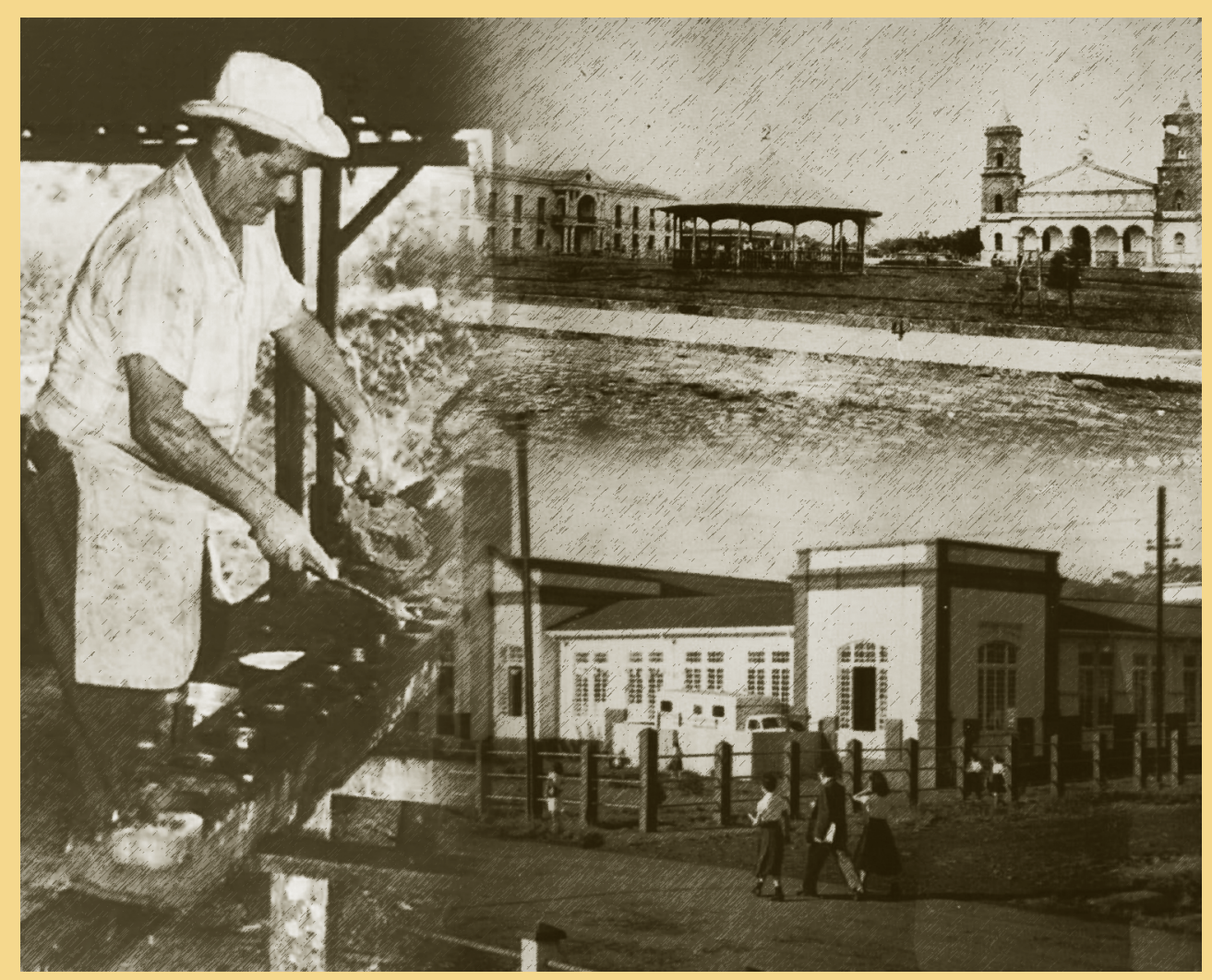

Director de la Revista: Dr. Juan José Marín Hernández juan.marinhernandez@ucr.ac.cr

Editor académico: Dr. David Díaz Arias - david.diaz@ucr.ac.cr

Editores invitados: M.Sc. William Solórzano Vargas - william.solorzano@ucr.ac.cr

M.Ed. Maynor Badilla Vargas - mainor.badilla@ucr.ac.cr

Editora técnica: M.Sc. Marcela Quirós G. - marcela.quiros@ucr.ac.cr 
Dr. Juan José Marín Hernández, Catedrático. Director del Centro de Investigaciones Históricas de América Central. Universidad de Costa Rica. Costa Rica. juan. marin@ucr.ac.cr

Dr. David Díaz Arias: Catedrático. Historia Política, Director del posgrado de Historia y Docente de la Escuela de Historia, Universidad de Costa Rica, Costa Rica.david.diaz@ucr.ac.cr

Dr. Ronny Viales Hurtado. Catedrático. Historia Económica y Social. Universidad de Costa Rica. Director de la Escuela de Historia. Costa Rica. ronny. viales@ucr.ac.cr

MSc. Francisco Enríquez. Historia Social. Universidad de Costa Rica. Costa Rica. francisco.enriquez@ucr. ac.cr

Dra. Ana María Botey. Historia de los movimientos sociales. Universidad de Costa Rica. Costa Rica. abotey@gmail.com

\section{Miembros del Consejo Asesor Internacional:}

Dr. José Cal Montoya. Universidad de San Carlos de Guatemala. Guatemala. jecalm@correo.url.edu.gt

Dr. Juan Manuel Palacio. Universidad Nacional de San Martín. Argentina.jpalacio@unsam.edu.ar

Dr. Eduardo Rey. Universidad de Santiago de Compostela. España. ereyt@usc.es

Dr. Heriberto Cairo Carou. Departamento de Ciencia Política y de la Administración III - Universidad Complutense de Madrid. España. hcairoca@cps.ucm.es

Dra. Rosa de la Fuente. Departamento de Ciencia Política y de la Administración III Universidad Complutense de Madrid. España. rdelafuente@cps. ucm.es

Dr. Javier Franzé. Departamento de Ciencia Política y de la Administración III Universidad Complutense de Madrid. España. javier.franze@cps.ucm.es

Dr. Jaime Preciado Coronado Departamento de Estudios Ibéricos y Latinoamericanos. Universidad de Guadalajara. México.japreco@hotmail.com

Dr. Gerónimo de Sierra. Vicerrector de la Universidade Federal da Integração Latino-Americana (UNILA) y Departamento de Sociología, Facultad de Ciencias

Sociales de la Universidad de la República. Uruguay. geronimo@fcs.edu.uy

Dr. Antonio Palazuelos. Departamento de Ciencia Política y de la Administración III - Universidad Complutense de Madrid. España. palazuelosa@cps. ucm.es

Dr. Werner Mackenbach. Universidad Potsdam. Alemania.werner.mackenbach@uni-potsdam.de

Dr. Guillermo Castro. Ciudad del Saber Panamá. Panamá.gcastro@cdspanama.org

Dra. Natalia Milanesio. University of Houston. Estados Unidos.nmilane2@Central.UH.EDU

Dr. Ricardo González Leandri. Consejo Superior de Investigaciones Científicas - España. España. rgleandri@gmail.com

Dra. Mayra Espina. Centro de Estudios Psicológicos y Sociológicos, La Habana. Cuba.mjdcips@ceniai.inf.cu

Dra. Montserrat Llonch. Departamento de Economía e Historia Económica Universidad Autónoma de Barcelona. España. montserrat.llonch@uab.es

Dra. Estela Grassi. Universidad de Buenos Aires. Argentina. estelagrassi@gmail.com

Dra. Yolanda Blasco. Universidad de Barcelona. España. yolandablasco@ub.edu

Dr. Alfredo Falero. Departamento de Sociología. Universidad de la República. Uruguay. alfredof@adinet. com.uy

Portada:

Fotografía: ( Collage "Campesinos en la producción de dulce en un trapiche de Rincón de Mora, Ran Ramón, 1987" ; "Panorámica del Antiguo Palacio Municipal, Antigua Iglesia, Parque y Kiosco de San Ramón, hacia 1920; y Escuela Jorge Washinton, San Ramón hacia 1955). Estas fotografías pertenecen a la Colección Fotográfica del Museo Regional de San Ramón- UCR. Montaje fotográfico: Lic. Juan Gabriel Madrigal Cubero ).

\section{Equipo Técnico Editorial:}

Diagramación y

Edición técnica:

M.Sc. Marcela Quirós Garita. marcela.quiros@ucr.ac.cr

Soporte técnico: Kevin Trejos Vargas

Revisión filológica: Baruc Chavarría Castro 
"Diálogos Revista Electrónica de Historia" se publica desde octubre de 1999.

\section{Diálogos está en los siguientes repositorios:}

Dialnet

http://dialnet.unirioja.es/servlet/

revista?tipo_busqueda=CODIGO\&clave_revista $=3325$

\section{Latindex}

http://www.latindex.unam.mx/larga.php?opcion=1\&folio=12995;

\section{UCRindex}

http://www.revistas.ucr.ac.cr

\section{Scielo}

http://www.scielo.cll

\section{eRevistas}

http://www.erevistas.csic.es/

\section{REDALYC}

http://redalyc.uaemex.mx/src/inicio/FrmBusRevs2.jsp?iEdoRev=2\&cvepai=11;

\section{LANIC}

http://lanic.utexas.edu/la/ca/cr/indexesp.html;

Repositorio de Revistas Universidad de Costa Rica

http://www.latindex.ucr.ac.cr/

Directorio y recolector de recursos digitales del

Ministerio de Cultura de España

http://roai.mcu.es/es/inicio/inicio.cmo

DOAJ Directory of open access \& Hybrid journals

http://www.doaj.org/doaj?func=byTitle\&hybrid=1\&query=D

Biblioteca de Georgetown

http://library.georgetown.edu/newjour/d/msg02735.htm

Asociación para el Fomento de los Estudios Históricos en Centroamérica

http://afehc.apinc.org/index.php?action=fi_aff\&id=1774

Universidad de Saskatchewan, Canadá

https://library.usask.ca/ejournals/view/1000000000397982

Monografias

http://www.monografias.com/Links/Historia/more12.shtml

\section{Hispanianova}

http://hispanianova.rediris.es/general/enlaces/hn0708.htm

Universidad del Norte, Colombia

http://www.uninorte.edu.co/publicaciones/memorias/enlaces.htm

Universidad Autónoma de Barcelona

http://seneca.uab.es/historia/hn0708.htm

Repositorio Invenia - Gestión del Conocimiento http://www.invenia.es/oai:dialnet.unirioja.es:ART0000086144

\section{Enlace Académico}

http://www.enlaceacademico.org/biblioteca/

revistas-en-formato-digital-centroamerica/

\section{Electronic Resources}

http://sunzi1.lib.hku.hk/ER/detail/hkul/3987318

Revistas académicas en texto completo http://web.prw.net/ vtorres/

Diálogos se anuncia en las siguientes instituciones y sitios académicos:

Maestroteca

http://www.maestroteca.com/detail/553/dialogos-revista-electronica-de-historia.html

Biblioteca de Georgetown

http://library.georgetown.edu/newjour/d/msg02735.htm

Asociación para el Fomento de los Estudios Históricos en Centroamérica

http://afehc.apinc.org/index.php?action=fi_aff\&id=1774

Universidad de Saskatchewan, Canadá

https://library.usask.ca/ejournals/view/1000000000397982

Monografias

http://www.monografias.com/Links/Historia/more12.shtm

Hispanianova

http://hispanianova.rediris.es/general/enlaces/hn0708.htm

Universidad del Norte, Colombia

http://www.uninorte.edu.co/publicaciones/memorias/enlaces.html

Universidad Autónoma de Barcelona

http://seneca.uab.es/historia/hn0708.htm

Repositorio Invenia - Gestión del Conocimiento

http://www.invenia.es/oai:dialnet.unirioja.es:ART0000086144

Enlace Académico

http://www.enlaceacademico.org/biblioteca/

revistas-en-formato-digital-centroamerical

\section{Electronic Resources}

http://sunzi1.lib.hku.hk/ER/detail/hkul/3987318

Revistas académicas en texto completo http://web.prw.net/ vtorres/

Diálogos Revista de Historia está catalogada por Sherpa Romeo como una revista verde.

La revista electrónica Diálogos es financiada por Vicerrectoría de Investigación de la Universidad de Costa Rica

Citado en: Scielo Dialnet - eRevistas - UCRindex Latindex - REDALYC - DOAJDirectorio y recolector de recursos digitales del Ministerio de Cultura de España

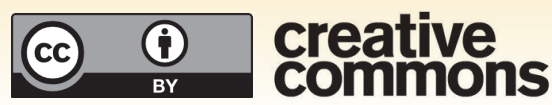




\title{
LOS DATOS CENSALES COMO FUENTE PARA LA CARACTERIZACIÓN DE LA PRODUCCIÓN AGROPECUARIA DEL CANTÓN DE PALMARES
} (1950-1984)

\section{CHARACTERIZATION OF THE AGRICULTURAL PRODUCTION IN PALMARES BASED ON CENSUSES DATA (1950-1984)}

\author{
Maynor Badilla Vargas \\ William Solórzano Vargas
}

\begin{abstract}
Palabras claves
Producción agropecuaria, Palmares, censos agropecuarios.

Keywords

Agricultural production, Palmares, agricultural censuses
\end{abstract}

Fecha de recepción: 21 de marzo, 2014 - Fecha de aceptación: 25 de junio, 2014

\begin{abstract}
Resumen
El siguiente trabajo consiste en un análisis esencialmente cuantitativo de la producción agropecuaria del cantón de Palmares, sustentado en estadísticas censales, correspondientes al periodo 1950-1984. Asimismo, se pretende aportar una lectura alternativa de la historia local, a partir de la historia agraria.
\end{abstract}

\begin{abstract}
The next document consists essentially on a quantitative analysis of the agricultural production in the Palmares County, which is based on censuses statistics located in the period within 19501984. By the same token it also pretends to provide an alternative reading about the local history constructed from the agrarian one.
\end{abstract}




\section{LOS DATOS CENSALES COMO FUENTE PARA LA CARACTERIZACIÓN DE LA PRODUCCIÓN AGROPECUARIA DEL CANTÓN DE PALMARES $(1950-1984)$}

\section{INTRODUCCIÓN}

Palmares es el cantón séptimo de la provincia de Alajuela y se ubica en el sector noroccidental del Valle Central de Costa Rica. Constituye un valle de $38.006 \mathrm{~km}^{2}$, que comenzó a ser poblado a partir de la década de 1830 por jóvenes colonos, que se fueron asentando en el territorio y paulatinamente desarrollaron diversas actividades agropecuarias, las cuales se constituyeron en el motor del desarrollo local, por lo menos hasta 1980.

A continuación se realiza un análisis a partir de diversas estadísticas censales, que contribuyen con la reconstrucción de la trayectoria de la producción agrícola de Palmares, correspondiente al periodo 1950-1984.

La estructura productiva local se ha caracterizado por la existencia de una serie de "ciclos productivos" (tabacalero, cafetalero y producción agrícola no tradicional). Con base en esta propuesta de periodización, se tiene que gran parte de los datos censales agropecuarios disponibles que se analizaron, giran en torno al cultivo del café, el cual tuvo un notorio auge a partir de la segunda mitad del siglo XX, generado por la caída de la producción tabacalera ${ }^{1}$, producto de la fuerte competencia - desleal- por parte de las compañías tabacaleras transnacionales, que entraron en el escenario de la producción de esta solanácea, así como las innovaciones tecnológicas que se incorporaron a la caficultura posterior a 1950. Es en este contexto que la actividad cafetalera se arraigó fuertemente en el cantón, denotando rasgos de "especialización" bastante significativos, pues el café llegó a ocupar en 1984 el 63\% de las tierras dedicadas a la producción agrícola del cantón, reduciendo el área agrícola destinada a otros cultivos como los granos básicos y la superficie dedicada a pastos para la ganadería.

\section{EVOLUCIÓN DEL ÁREA AGRÍCOLA}

Para iniciar el análisis de la estructura productiva local, la Tabla 1 muestra, en primera instancia, la evolución del área agrícola del cantón, durante el periodo 19501984. Aquí se puede apreciar cómo de 1950 a 1963 hubo un incremento sostenido en el área utilizada en la agricultura, aumento que se hace muy evidente en los cultivos permanentes, especialmente en la actividad cafetalera. Lo anterior motivado por la gran demanda de estos productos a nivel internacional, gracias al crecimiento y expansión que presentaba la economía mundial de la posguerra. Para el periodo 1963- 
1973, la actividad agropecuaria en Palmares, se estanca e inclusive se aprecia una leve disminución hacia el año 1973. Pero de esta fecha en adelante, se evidenció un importante descenso en el área agrícola total, pasando de 5.103 hectáreas en el año de 1973, a 3.578 hectáreas en 1984, lo que representa un descenso del área agrícola del orden del $30 \%$, en un periodo de once años.

En relación con el número de unidades productivas, su comportamiento fue similar al del área agrícola, especialmente la tendencia descendente que se dio a partir de 1973. Durante el periodo intercensal correspondiente a los años 1950-1973, las unidades productivas se incrementaron en más del $100 \%$, pero decayeron en un $9 \%$ durante los años 1973-1984.

Este aumento en el número de unidades productivas en el periodo 1950-1973, no parece ser producto de la apertura de nuevas tierras para el cultivo, sino, más bien, refleja un acelerado proceso de fragmentación de las unidades productivas (la división de las propiedades para heredarlas a los hijos parece ser el factor explicativo de este proceso) que tuvo lugar a partir del año 1950, y que aumentó de 1963 en adelante, tendencia que se mantuvo hasta mediados de 1970.

La idea anterior se ve reafirmada si se analiza el indicador de tamaño de las fincas. El área promedio se redujo, pasando de 6,1 hectáreas, en 1950, a 4 hectáreas, en 1984; lo que se tradujo en un descenso del 35\% del área promedio de las fincas del cantón, durante el periodo 1950-1984. Esto también refleja lo limitado de las unidades productivas en cuanto a su área; de ahí que muchas se caracterizaron por su explotación intensiva, así como por su alto grado de "especialización cafetera".

\section{USO DEL SUELO}

En relación con el uso del suelo, hubo predominio de las tierras dedicadas a cultivos permanentes, especialmente al café, seguidas de las destinadas a pastos y labranza (o cultivos de subsistencia) y, por último, las ocupadas por bosques y charrales. De la información proporcionada por la Tabla 2, se extrae una clara conclusión: el uso del suelo en el cantón de Palmares, durante el periodo 1955-1984, experimentó una significativa transformación, dado que las áreas destinadas a actividades agrícolas, en su conjunto, se redujeron considerablemente para el año 1984.

Esto refleja, claramente, la presión por habilitar nuevas tierras para otros usos, especialmente el urbano, el cual, como se ha indicado, obedeció al notable crecimiento demográfico del cantón y a factores externos como la crisis económica internacional de la década de 1970 y la posterior caída de los precios del café en el mercado extranjero.

Con el propósito de ilustrar este fenómeno, se adjuntan algunos datos que corroboran esta tendencia, la cual es muy marcada durante el periodo 1973-1984. Por 


\section{Tabla 1}

PALMARES ÁREA AGRÍCOLA, NÚMERO DE UNIDADES

PRODUCTIVASY ÁREA PROMEDIO DE LAS FINCAS,

EN HECTÁREAS* (1950-1984)

\begin{tabular}{lccccc}
\hline \multicolumn{1}{c}{ INDICADORES } & \multicolumn{5}{c}{ AÑOS CENSALES } \\
\hline & 1950 & 1955 & 1963 & 1973 & 1984 \\
& 3.032 & 4.585 & 5.050 & 5.103 & 3.578 \\
Área agrícola total Ha & 494 & 557 & 668 & 894 & 888 \\
$\mathrm{~N}^{\text {o unid. prod. cantonal }}$ & & & & & \\
& 6,1 & 8,23 & 7,55 & 5,70 & 4,02 \\
\hline
\end{tabular}

*Nota: Si bien en los censos de 1950, 1955, 1963 y 1973 el área destinada a cada cultivo está dado en manzanas, en el censo de 1984 los datos se presentaron en hectáreas. Por tal razón y para poder presentar de manera homogénea la información en los diferentes cuadros que elaboramos, decidimos trabajar en hectáreas, y se convirtieron las manzanas en hectáreas multiplicando el número de manzanas por 0.69; a pesar de que la cifra correcta para tal conversión es 0.6989. Fuente: Elaboración propia a partir de: (Dirección General de Estadísticas y Censos, 1953; Dirección General de Estadísticas y Censos, 1959; Dirección General de Estadísticas y Censos, 1964; Dirección General de Estadísticas y Censos, 1974; Dirección General de Estadísticas y Censos, 1987).

ejemplo, en lo que respecta a cultivos permanentes, hubo un descenso del 13\% en su área, durante los años indicados; el área cultivada de pastos, para este mismo periodo, se redujo en un $62 \%$, aproximadamente, (ver Figura 1).

El régimen de tenencia de la tierra para el cantón de Palmares, durante el periodo 1950-1984 (Tabla 3), se caracterizó por el predominio de unidades productivas propias, las cuales representaban, para 1984, un total de 3.480 hectáreas, lo que equivalía al 97\% del total de extensión de las fincas para ese año. Una situación similar se dio en los periodos anteriores (1950, 1955, 1963 y 1973), de manera tal que esta forma de tenencia de la tierra - la propia - fue la preponderante sobre las restantes. Esto, sin lugar a dudas, constituye un elemento histórico que se mantiene hasta la actualidad, el cual consiste en la distribución equitativa de la tierra en el cantón de Palmares, debido en gran medida al fraccionamiento de esta, por ejemplo, vía herencia. Al respecto Jiménez destaca que: 
Tabla 2

PALMARES

USO DEL SUELO, EN HECTÁREAS, (1955-1984)

\begin{tabular}{lcccc}
\hline \multicolumn{1}{c}{ INDICADORES } & \multicolumn{4}{c}{ AÑOS CENSALES } \\
\hline & 1955 & 1963 & 1973 & 1984 \\
\hline Cultivos permanentes & 1.493 & 2.262 & 2.386 & 2.116 \\
Labranza & 484 & 451 & 326 & 263 \\
Pastos & 2.417 & 2.168 & 1.692 & 1.045 \\
Bosques & 201 & 72 & 617 & 64 \\
Charrales & 109 & 115 & 60 & 30 \\
Otras tierras & 3 & 29 & 20 & 57 \\
\hline
\end{tabular}

*Nota: El año 1950 no se incluyó, debido a no disponer de la información necesaria. Fuente: Elaboración propia a partir de: (Dirección General de Estadísticas y Censos, 1959; Dirección General de Estadísticas y Censos, 1964; Dirección General de Estadísticas y Censos, 1974; Dirección General de Estadísticas y Censos, 1987).

...esta zona se caracteriza, desde décadas anteriores al desarrollo de la actividad del café, por una estructura de tenencia de la tierra mucho más equitativa que la de otras localidades del país; por lo que la presencia de un campesinado medio y pobre con tierra es un fenómeno que data desde la colonización de la zona. (1985, p. 22).

\section{Además,}

...el desarrollo de la actividad del café no produce profundas modificaciones en lo que respecta al proceso de concentración de la tierra: y que, hasta cierto punto, la presencia del café y su desarrollo en los últimos treinta años, contribuye al desarrollo y consolidación de la pequeña y mediana producción. (1985, p. 23). 


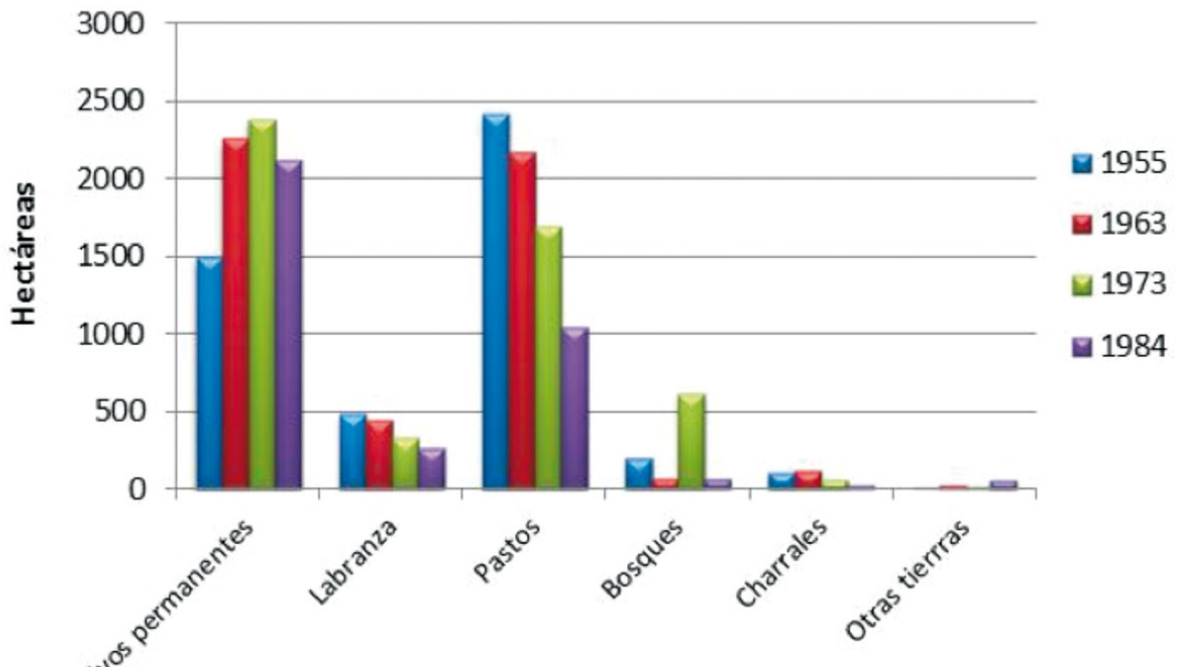

\section{Uso del suelo}

Figura 1. Palmares Uso del suelo, en hectáreas, (1955, 1963, 1973 y 1984).

Nota: El año 1950 no se incluyó, debido a no disponerse de la información necesaria. Fuente: Elaboración propia a partir de: (Dirección General de Estadísticas y Censos, 1959; Dirección General de Estadísticas y Censos, 1964; Dirección General de Estadísticas y Censos, 1974; Dirección General de Estadísticas y Censos, 1987).

Tabla 3

PALMARES RÉGIMEN DE TENENCIA DE LA TIERRA, EN HECTÁREAS, (1950-1984)

\begin{tabular}{lccccc}
\hline \multicolumn{1}{l}{$\begin{array}{c}\text { FORMAS DE } \\
\text { TENENCIA } \\
\text { DE LA TIERRA }\end{array}$} & 1950 & 1955 & 1963 & 1973 & 1984 \\
\hline Propia & 2.856 & 4.009 & 4.408 & 4.870 & 3.480 \\
Arrendada & 90 & 20 & 16 & 229 & 75 \\
Esquilmo & s.d. & 5 & 1,5 & s.d. & 0 \\
Propio y arrendado & s.d. & 192 & 139 & s.d. & 59 \\
Propio y esquilmo & s.d. & 36 & 381 & s.d. & 14 \\
Otras formas & 85 & 32 & 75 & 4 & 102 \\
\hline
\end{tabular}

Nota: Fuente: Elaboración propia a partir de: (Dirección General de Estadísticas y Censos, 1953; Dirección General de Estadísticas y Censos, 1959; Dirección General de Estadísticas y Censos, 1964; Dirección General de Estadísticas y Censos, 1974; Dirección General de Estadísticas y Censos, 1987). 
La Figura 2 ilustra la idea anteriormente comentada; en él se puede apreciar cómo, a lo largo de todo el periodo analizado, la forma de tenencia de la tierra predominante en este cantón fue la propia. Es decir, una característica básica de la comunidad palmareña fue el hecho de que sus pobladores, en su gran mayoría, eran propietarios directos de los terrenos en los que producían.

\section{LA PRODUCCIÓN AGRÍCOLA LOCAL: ESPECIALIZACIÓN HACIA EL CULTIVO DEL GRANO DE ORO}

El análisis de la producción agrícola del cantón inicia con el cultivo del café, el cual, durante el periodo 1950-1955 asumió un carácter de "especialización"; no obstante, esta tendencia se manifestaba ya desde 1935, año en el cual se registraron 1.326 hectáreas cultivadas de café ${ }^{2}$.

Es importante destacar que el área cantonal destinada al cultivo del café experimentó un constante crecimiento durante el periodo 1950-1984. En 1950, el café ocupaba el $34 \%$ de la totalidad de las tierras destinadas a cultivos del cantón; para el año 1955, el 31\%; en 1963, el 43\%; en 1973, el 47\% y, según el último censo

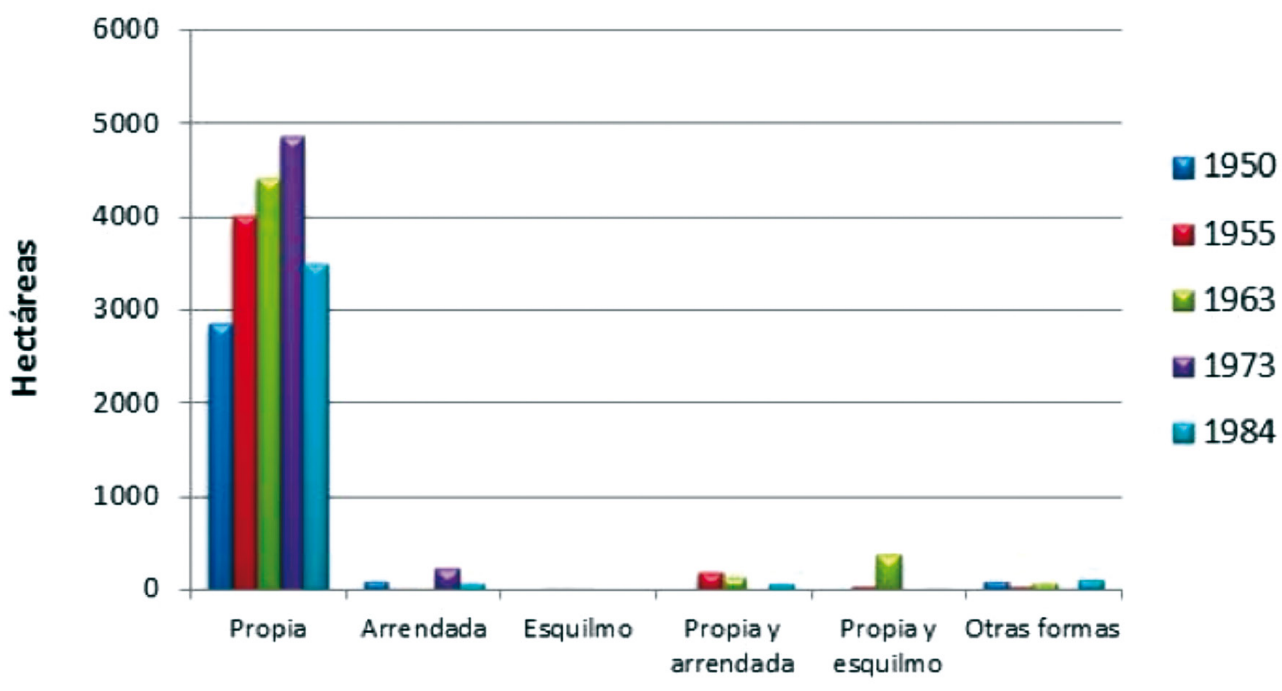

Formas de tenencia de la tierra

Figura 2. Palmares. Régimen de tenencia de la tierra, en hectáreas, (1950, 1955, 1963, 1973 y 1984) Fuente: Elaboración propia a partir de: (Dirección General de Estadísticas y Censos, 1953; Dirección General de Estadísticas y Censos, 1959; Dirección General de Estadísticas y Censos, 1964; Dirección General de Estadísticas y Censos, 1974; Dirección General de Estadísticas y Censos, 1987). 
agropecuario (1984), el café ocupaba el 63\% del total de las tierras de cultivo del cantón de Palmares. Sin embargo, a partir de 1973 tuvo lugar una reducción del 11\% de la superficie cultivada de café, pasando de 2.381 hectáreas, en 1973, a 2.149 en el año 1984.

Con respecto a los rendimientos por hectárea, la Tabla 4 muestra que estos experimentaron un crecimiento de casi un $300 \%$, durante el periodo en análisis; pasando de 6,7 fanegas por hectárea en 1950, a 26,2 fanegas por hectárea en 1984. La producción, por su parte, mostró un incremento sostenido, pasando de 6.894 fanegas en 1950, a 56.320 en 1984, lo que representó un aumento del 716\%, en un lapso de 34 años.

Estos incrementos, en la producción y en los rendimientos, fueron generados por diferentes factores, entre ellos destacan: la habilitación de mayor cantidad de tierras destinadas al cultivo del café, como consecuencia del decaimiento de la actividad tabacalera; la paulatina incorporación, por parte de los productores locales, de algunas innovaciones tecnológicas derivadas de la Revolución Verde, especialmente el cultivo de nuevas variedades de café. El carácter intensivo y de especialización que asumió la caficultura en Palmares contribuyó para que la producción y los rendimientos manifestaran un crecimiento importante durante el periodo 1950-1984.

El cultivo de granos básicos constituyó una actividad poco significativa desde el punto de vista comercial; sin embargo, para el consumo familiar fue de gran

\section{Tabla 4}

PALMARES, ÁREA CULTIVADA (EN HECTÁREAS), NÚMERO DE UNIDADES PRODUCTIVAS, PRODUCCIÓN Y RENDIMIENTOS DEL CAFÉ, (1950- 1984)

\begin{tabular}{ccccc}
\hline $\begin{array}{c}\text { AÑO } \\
\text { CENSAL }\end{array}$ & $\begin{array}{c}\text { ÁREA CULTIVADA } \\
\text { (HECTÁREAS) }\end{array}$ & $\begin{array}{c}\text { No UNIDADES }^{\circ} \\
\text { PRODUCTIVAS }\end{array}$ & $\begin{array}{c}\text { PRODUCCIÓN } \\
\text { (FANEGAS) }\end{array}$ & $\begin{array}{c}\text { RENDIMIENTOS } \\
\text { (FANEGAS/HA) }\end{array}$ \\
\hline 1950 & 1.028 & 376 & 6.894 & 6,7 \\
1955 & 1.399 & 511 & 15.092 & 10,7 \\
1963 & 2.162 & 621 & 38.152 & 17,6 \\
1973 & 2.381 & 792 & 48.909 & 20,5 \\
1984 & 2.149 & 761 & 56.320 & 26,2 \\
\hline
\end{tabular}

Nota: Elaboración propia a partir de: (Dirección General de Estadísticas y Censos, 1953; Dirección General de Estadísticas y Censos, 1959; Dirección General de Estadísticas y Censos, 1964; Dirección General de Estadísticas y Censos, 1974; Dirección General de Estadísticas y Censos, 1987). 


\section{Tabla 5}

PALMARES, ÁREA CULTIVADA (EN HECTÁREAS), NÚMERO DE UNIDADES PRODUCTIVAS, PRODUCCIÓN Y RENDIMIENTOS DEL CULTIVO DEL FRIJOL, (1955-1984)

\begin{tabular}{ccccc}
\hline AÑO CENSAL & $\begin{array}{c}\text { ÁREA CULTIVADA } \\
\text { (EN HECTÁREAS) }\end{array}$ & $\begin{array}{c}\mathrm{N}^{\circ} \text { UNIDADES } \\
\text { PRODUCTIVAS }\end{array}$ & PRODUCCIÓN (KG) & $\begin{array}{c}\text { RENDIMIENTOS } \\
\text { (KG/HA) }\end{array}$ \\
\hline 1955 & 203 & 254 & 41.100 & 201,89 \\
1963 & 187 & 232 & 42.437 & 219,31 \\
1973 & 116 & 170 & 46.974 & 402,17 \\
1984 & 77 & 143 & 37.833 & 486,28 \\
\hline
\end{tabular}

Fuente: Elaboración propia a partir de: (Dirección General de Estadísticas y Censos, 1959; Dirección General de Estadísticas y Censos, 1964; Dirección General de Estadísticas y Censos, 1974; Dirección General de Estadísticas y Censos, 1987).

importancia. Los datos que aporta la Tabla 5 reflejan la escasa área destinada para el cultivo de frijol. Por ejemplo, para el año 1955, este grano ocupaba, únicamente, el 4,29\% del total de la superficie de las fincas; para 1963, el 3,70\%; en 1973, el $2,29 \%$ y, para el año 1984, el mismo porcentaje $(2,29 \%)$.

En el caso del maíz (Tabla 6), la situación es bastante similar. A lo largo de todo el periodo (1955-1984) se aprecia una disminución cada vez más significativa, tanto en el área cultivada como en el número de unidades productivas que practicaban el cultivo de esta gramínea. Por ejemplo, para el año 1955, este grano ocupaba el 6,91\% del total de la superficie de las fincas; en 1963, el 5,08\%; para el año 1973, el 3,37\%, y en 1984, el 2,69\%.

Con respecto a la producción, ambas actividades presentaron un descenso importante. Durante el periodo 1955-1984, la producción de frijol se redujo en un $8 \%$, mientras que la de maíz, para el mismo periodo, se redujo en un 54\%. No obstante, a pesar de este descenso en la producción, los rendimientos ( $\mathrm{kg} / \mathrm{ha})$, presentaron un comportamiento distinto. Por ejemplo, los rendimientos de frijol, para este periodo, se incrementaron en un $141 \%$ y los de maíz en un $64 \%$. Todo ello, a pesar de la paulatina reducción del área destinada al cultivo de estos granos básicos. Esta situación sugiere que se aplicaron mejores técnicas agrícolas y una mayor intensificación de estos cultivos, dando como resultado un incremento de los rendimientos por hectárea. 


\section{Tabla 6}

PALMARES, ÁREA CULTIVADA (EN HECTÁREAS), NÚMERO DE UNIDADES PRODUCTIVAS, PRODUCCIÓN Y

RENDIMIENTOS DEL CULTIVO DEL MAÍZ, (1955-1984)

\begin{tabular}{ccccc}
\hline $\begin{array}{c}\text { AÑOS } \\
\text { CENSALES }\end{array}$ & $\begin{array}{c}\text { ÁREA CULTIVADA } \\
\text { (HECTÁREAS) }\end{array}$ & $\begin{array}{c}\mathrm{N}^{\circ} \text { UNIDADES } \\
\text { PRODUCTIVAS }\end{array}$ & PRODUCCIÓN (KG) & RENDIMIENTOS (KG/HA) \\
\hline 1955 & 327 & 342 & 239.750 & 731,27 \\
1963 & 256 & 282 & 323.500 & $1.218,13$ \\
1973 & 171 & 251 & 223.463 & $1.299,95$ \\
1984 & 91 & 181 & 109.239 & $1.197,79$ \\
\hline
\end{tabular}

Nota: Elaboración propia a partir de: (Dirección General de Estadísticas y Censos, 1959; Dirección General de Estadísticas y Censos, 1964; Dirección General de Estadísticas y Censos, 1974; Dirección General de Estadísticas y Censos, 1987).

\section{LA ACTIVIDAD GANADERA}

La actividad ganadera (Tabla 7), sin lugar a dudas, no ha sido el fuerte de la economía palmareña. Esto lo constata la escasa cantidad de cabezas de ganado reportadas por los censos agropecuarios de los años 1950, 1955, 1963, 1973 y 1984. En el de 1950, solamente se reportaron 1.930 cabezas de ganado vacuno y para el año 1984, 2.048 cabezas. De los datos de los cincos censos agropecuarios se obtiene un promedio para el periodo 1950-1984, de 2.264 cabezas de ganado vacuno, para el cantón de Palmares. El año censal que reporta la mayor cantidad de reses corresponde a 1955, con 2.785, y el que menos reportó fue el de 1950, con 1.930 cabezas de ganado vacuno.

La mayor cantidad de unidades productivas, dedicadas a la ganadería, corresponden al año 1955, iniciándose a partir de esta fecha un descenso en la cantidad de fincas ganaderas, como lo indican los datos del año 1984, en el que aparecen 136 explotaciones dedicadas a la ganadería vacuna. Esto equivale a una reducción del $111 \%$ en la cantidad de unidades productivas, para este último año. A pesar de estos datos (reducción de cabezas de ganado y de unidades productivas), la carga animal reveló un incremento, pasando de 1,1 cabezas de ganado por hectárea, en 1955, a 1,9 en el año 1984. Esto implica que 
los productores ganaderos intensificaron su actividad, explotando de manera más efectiva el espacio disponible, o bien, muchos de ellos optaron por convertirse en cafetaleros, relegando a un segundo plano la ganadería o desechándola del todo.

\section{Tabla 7}

SUPERFICIE DE PASTOS (EN HECTÁREAS), NÚMERO DE UNIDADES PRODUC-

TIVAS, CABEZAS DE GANADO Y CARGA ANIMAL, (1950-1984)

\begin{tabular}{ccccc}
\hline $\begin{array}{c}\text { AÑOS } \\
\text { CENSALES }\end{array}$ & $\begin{array}{c}\text { PASTOS (EN } \\
\text { HECTÁREAS) }\end{array}$ & $\begin{array}{c}\mathrm{N}^{\circ} \text { UNIDADES } \\
\text { PRODUCTIVAS }\end{array}$ & $\begin{array}{c}\text { CABEZAS DE } \\
\text { GANADO }\end{array}$ & $\begin{array}{c}\text { CARGA } \\
\text { ANIMAL }\end{array}$ \\
\hline 1950 & 1.115 & 287 & 1.930 & 1,7 \\
1955 & 2.417 & 290 & 2.785 & 1,1 \\
1963 & 2.168 & 252 & 2.532 & 1,1 \\
1973 & 1.692 & 209 & 2.025 & 1,1 \\
\hline 1984 & 1.045 & 136 & 2.048 & 1,9 \\
\hline
\end{tabular}

Nota: Elaboración propia a partir de: (Dirección General de Estadísticas y Censos, 1953; Dirección General de Estadísticas y Censos, 1959; Dirección General de Estadísticas y Censos, 1964; Dirección General de Estadísticas y Censos, 1974; Dirección General de Estadísticas y Censos, 1987).

\section{CONCLUSIONES}

A manera de síntesis, el análisis de estos datos muestra que la actividad agropecuaria en Palmares, durante el periodo 1950-1984, presentó las siguientes características:

De 1950 a 1973, el área utilizada en la agricultura experimentó un crecimiento sostenido, especialmente en los cultivos permanentes y dentro de estos, el cultivo de café fue el que más creció, tanto en la cantidad de hectáreas sembradas, como en producción y productividad por hectárea. Fue tal el crecimiento de la actividad cafetalera en el cantón, que el cultivo de este grano redujo de manera significativa el área destinada a otros cultivos como el tabaco, los granos básicos (frijol y maíz) y tierras de repasto dedicadas a la ganadería, pues muchas fueron convertidas en cafetales. Lo que lleva a afirmar que durante el periodo descrito, en Palmares se produjo una tendencia hacia la "especialización" en la producción cafetalera.

Este auge en el desarrollo cafetalero del cantón se vio impulsado por varios factores. Por un lado, la actividad tabacalera, muy tradicional en este cantón, 
desde finales del siglo XIX y la primera mitad del siglo XX, se vio seriamente amenazada por la entrada en escena de las compañías transnacionales, que empezaron a controlar la producción y comercialización de este producto y sus derivados. Por otro lado, el denominado "grano de oro", además de ser un cultivo muy apto agroecológicamente para esta zona, experimentaba una gran demanda en el mercado internacional en el periodo de posguerra y era muy rentable, dados los altos precios en que se lograba vender dicho producto.

Durante el periodo 1973-1984, los datos muestran un estancamiento en la actividad agropecuaria desde inicios de 1970 y una disminución sostenida y sistemática en el área dedicada a la agricultura, del orden del 30\% al finalizar este periodo. Indudablemente, la crisis económica generada por los altos precios del petróleo a nivel internacional en la década de los años setenta, tuvo su impacto en las actividades productivas de las comunidades rurales del país. Además de esta causa, hubo otros factores que impactaron la actividad agropecuaria del cantón, entre ellos: el acelerado proceso de urbanización que restó tierra para las actividades agrícolas, el incremento en el precio de los insumos, el aumento del costo de la mano de obra, debido a su escasez, y la disminución del precio del café en términos reales.

\section{CITAS Y NOTAS}

1. En relación con la producción tabacalera, Alfaro y Badilla (2010), realizan una síntesis sobre la trayectoria histórica de este cultivo en el cantón de Palmares, desde la década de los años treinta del siglo XIX, hasta la década de 1960. Asimismo, se refieren a aspectos generales de la historia del tabaco en Costa Rica y a las especificidades de esta solanácea en la comunidad, enfatizando en el desarrollo cooperativo que se gestó debido a su cultivo, procesamiento y comercialización.

2. (Peters y Rojas, 2010)

\section{REFERENCIAS}

Alfaro A. y Badilla, M. (2010). Apuntes sobre la trayectoria del tabaco en el cantón de Palmares. Revista Herencia, 23(1), pp.113-127.

Dirección General de Estadísticas y Censos. (1953).Censo Agropecuario de 1950, Ministerio de Economía y Hacienda. Costa Rica: Imprenta Instituto Geográfico. 
Dirección General de Estadísticas y Censos. (1959). Censo Agropecuario de 1955, Ministerio de Economía y Hacienda. Costa Rica: Impreso D.G.E.C.

Dirección General de Estadísticas y Censos. (1964). Censo Agropecuario de 1963, Ministerio de Economía y Hacienda. Costa Rica: Impreso D.G.E.C.

Dirección General de Estadísticas y Censos. (1974). Censo Agropecuario de 1973, Ministerio de Economía, Industria y Comercio. Costa Rica: Impreso D.G.E.C. Sección Publicaciones.

Dirección General de Estadísticas y Censos. (1987). Censo Agropecuario de 1984, Ministerio de Economía, Industria y Comercio. Costa Rica: Imprenta Nacional.

Jiménez, A.C. (1985). Participación del campesinado parcelario en la producción del café en Costa Rica: 1950-1980. Análisis de caso: cantón de Palmares de la provincia de Alajuela. (Tesis de Maestría). Universidad Nacional Autónoma de México, México.

Peters, G. y Rojas, M. (2010). El Censo Cafetalero Costarricense, 1935. Disponible en: http://www. historia.una.ac.cr/index.php?option=com_remository\&Itemid=56\&func=startdown $\& \mathrm{id}=71$

\section{ACERCA DE LOS AUTORES}

Maynor Badilla Vargas: Profesor e investigador, Sede de Occidente, Universidad de Costa Rica. Correo electrónico: mainor.badilla@ucr.ac.cr

William Solórzano Vargas: Profesor e investigador, Sede de Occidente, Universidad de Costa Rica. Correo electrónico: wsolorzanovargas@yahoo.es 